\title{
Optimal energy systems design applied to an innovative ocean-wind energy converter
}

\author{
M. Holl ${ }^{1}$, M. Platzer ${ }^{2} \&$ P. Pelz ${ }^{1}$ \\ ${ }^{1}$ Chair of Fluid Systems, Technische Universität Darmstadt, Germany \\ ${ }^{2}$ AeroHydro Research \& Technology Associates, USA
}

\begin{abstract}
System level optimization is used to design an innovative ocean-wind energy converter to meet the "as good as it can be done" design objective. This general design procedure is then applied to the design of a $20 \mathrm{~kW}$ energy system and it is demonstrated that a combined energetic and economical design procedure is required for an optimal solution.

Keywords: techno-economical optimization, energy ship, renewable energy, hydrogen production, Pareto-frontier.
\end{abstract}

\section{Introduction}

Most of the planet's renewable energy is to be found in the winds over vast ocean areas. Therefore innovative concepts are needed to tap into this energy reservoir. One possible concept involves the operation of wind-propelled vessels equipped with hydrokinetic turbines so that the kinetic energy of the water flow relative to the hydrokinetic turbine is converted into electricity. This electric power then is used to split sea water electrolytically into hydrogen and oxygen. The hydrogen gas then is compressed and stored in tanks. Although the basic concept seems to have been first proposed by Salomon [1] as long ago as 1982 the first more quantitative analyses have been presented only during the past five years by Platzer et al. [2-4] and Kim and Park [5].

\section{System analysis and optimization}

The energy converting system can be physically modelled and described through a simple momentum balance [6]. In this balance, the thrust force of the sails is in 
a mechanical equilibrium with the resistance force of the vessel and the turbine. The turbine resistance force can be controlled through the axial induction factor. The axial induction factor $\zeta$ quantifies the deceleration of the water current and therefore is a measure of the extracted kinetic energy. The result of this force balance is a term for the dimensionless vessel speed $v:=V / c$ with the vessel speed $V$ and the absolute wind velocity $c$

with the substitution

$$
v_{1 / 2}=q \cos \alpha_{0} \pm \sqrt{q^{2} \cos ^{2} \alpha_{0}-q}
$$

$$
q:=\frac{\sin \beta c_{L} \varrho}{\sin \beta c_{L} \varrho-c_{D} a_{V}-\left(1-\zeta^{2}\right) a_{T}}<0 .
$$

Only the positive solution is physically reasonable. With reference to wind turbines, the dimensionless vessel speed can be interpreted as the tip speed ratio. The angles $\alpha_{0}$ and $\beta$ define the inflow at the sail. $c_{L}$ denotes the lift coefficient and $c_{D}$ the drag coefficient of the vessel. The dimensionless area of the vessel is defined by $a_{V}:=A_{V} / A$ with the vessel area $A_{V}$ and the sail area $A$. The dimensionless turbine area is defined as $a_{T}:=A_{T} / A$ with the turbine area $A_{T}$. The change in working fluids is acounted for by the dimensionless density ratio $\varrho:=\varrho_{g} / \varrho_{l}$, where $\varrho_{g}$ denotes the air density and $\varrho_{l}$ the water density. The defined dimensionless vessel speed has to match to the dimensionless vessel speed $v_{V}$, defined by the velocity triangle at the sail section. Through the equality of these vessel speeds an expression for $\zeta$ is found

$$
\zeta=\sqrt{\frac{\sin \beta c_{L} \varrho}{a_{T}}\left(\frac{2 v_{V} \cos \alpha_{0}-1}{v_{V}^{2}}-1\right)+\frac{c_{D} a_{V}}{a_{T}}+1}
$$

After the physical modeling, the system is described and optimized under the aspects of energy and economy. A block diagram of the energy ship is shown in Figure 1.

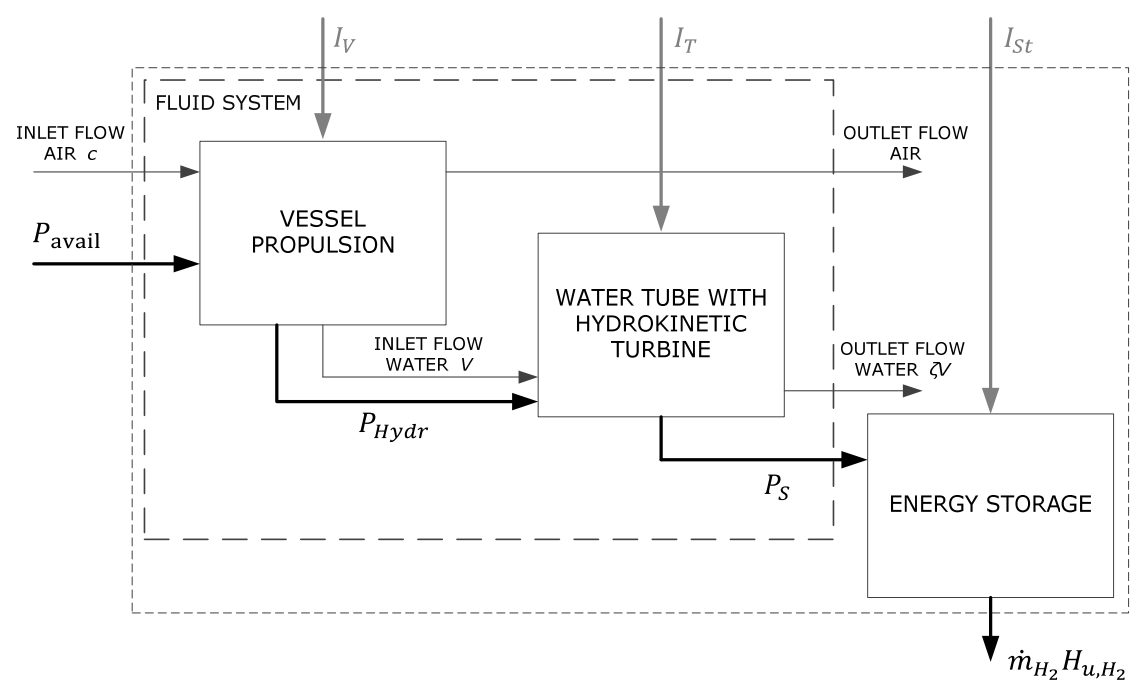

Figure 1: Block diagram of the energy ship. 
The flow velocities of the two working fluids, air and water, are shown in the Figure. Also the flow of energy through the system is depicted. It can be found that the available power $P_{\text {avail }}$ is the reference power. The hydraulic power $P_{H y d r}=T * V$, with the thrust $T$, is fed to the hydrokinetic turbine. This hydraulic power is than partially converted into the turbine power $P_{S}$ which is fed into the electrolyser. The chemical power of hydrogen can be calculated as $\dot{m}_{\mathrm{H}_{2}} \mathrm{H}_{u, \mathrm{H}_{2}}$, where $\dot{m}_{\mathrm{H}_{2}}$ denotes the mass flow of hydrogen and $H_{u, H_{2}}$ the caloric value of hydrogen. To describe the system economically the investment costs of all system components are shown in Figure 1. They include the investment cost of the vessel $I_{V}$, the turbine $I_{T}$ and the storage technology $I_{s t}$. Operation and maintenance costs are neglected in this first approach. The economical revenue can be calculated as the amount of hydrogen that can be sold.

To describe and optimize the system with respect to energy, a system quantity needs to be defined that addresses the energetic efficiency. According to the work of Betz for wind turbines [7] this quantity is the coefficient of performance $C_{P}:=P_{S} / P_{\text {avail }}$, defined as the ratio of the turbine power and the available power. As a result of the first law of thermodynamics the turbine power is $P_{S}=\eta \Delta p A_{T} \bar{V}$ with the arithmetic average velocity $\bar{V}=V(1+\zeta) / 2$. Using the previously defined dimensionless quantities the coefficient of performance is

$$
\frac{C_{P}}{\eta}=\frac{1}{\varrho} v^{3} a_{T} \frac{1+\zeta}{2}\left(1-\zeta^{2}\right) .
$$

The economic description is accomplished by applying the economic profit function to the energy system. In its simplest form the economic profit function is

$$
G=R-C
$$

where $G$ denotes the profit, $R$ the revenue and $C$ the costs. The profit, revenue and costs per year then are denoted by $\dot{G}=\dot{R}-\dot{C}$. The costs can be calculated as the sum of all investments. Furthermore, the discounting of the capital needs to be considered. Therefore an annuity loan is assumed where in a series of $n$ yearly constant payments the capital and the interest of capital is repaid. The yearly payment can be calculated as

$$
\dot{C}=\operatorname{CRF}\left(I_{V}+I_{T}+I_{S t}\right) .
$$

The sum of all investments is the capital which is multiplied with the capital recovery factor $C R F$. The capital recovery factor is defined as

$$
C R F=\frac{i(1+i)^{n}}{(1+i)^{n}-1}
$$

with the rate of interest $i$ and the amount of yearly payments $n$. By implementing the area specific prices $p_{V}:=I_{V} / A_{V}, p_{T}:=I_{T} / A_{T}$ and $p_{S t}:=I_{S t} / A$ the yearly costs can be calculated as

$$
\dot{C}=\operatorname{CRF}\left(p_{V} A_{V}+p_{T} A_{T}+p_{S t} A\right) .
$$


The area related price of the storage technology has been related to the sail area, because the capacity of the storage technology is proportional to the turbine power and therewith to the sail area. The revenue can be calculated as

$$
\dot{R}=\dot{m}_{H_{2}} p_{H_{2}},
$$

where $p_{\mathrm{H}_{2}}$ denotes the price of hydrogen per kilogram. Through the efficiency factor of the electrolyser $\eta_{\text {Elec }}:=\dot{m}_{\mathrm{H}_{2}} H_{u, H_{2}} / P_{S}$ the revenue can be calculated as

$$
\dot{R}=P_{S} \frac{\eta_{G e n} \eta_{\text {Elec }}}{H_{u, H_{2}}} p_{H_{2}} \text {. }
$$

With this equation a coupling of the economic and energetic system quantities is obtained. Through the definition of the coefficient of performance the turbine power can be calculated as $P_{S}=P_{\text {avail }} C_{P}$, which leads to the final estimation of the revenue

$$
\dot{R}=\frac{\varrho g}{2} c^{3} A C_{P} \frac{\eta_{E l e c} \eta_{G e n}}{H_{u, H_{2}}} p_{H_{2}}
$$

with the available power $P_{\text {avail }}=\varrho_{g} c^{3} A / 2$. Finally the sail area related profit function per year is

$$
\begin{gathered}
\frac{\dot{G}}{A}=\frac{\varrho_{g}}{2} c^{3} C_{P} \frac{\eta_{\text {Elec }} \eta_{G e n}}{H_{u, H_{2}}} p_{H_{2}}+ \\
-\operatorname{CRF}\left(p_{V} a_{V}+p_{T} a_{T}+p_{S t}\right)
\end{gathered}
$$

With this equation the energetic system quantity and the economic system quantity are combined. By combining those quantities in a single function a Pareto- optimization is possible. The aim of the optimization is to maximize the yearly profit. The optimization requirements can be summarized to

$$
\left(\begin{array}{c}
\frac{\partial}{\partial a_{T}}\left(\frac{\dot{G}}{A}\right) \\
\frac{\partial}{\partial a_{V}}\left(\frac{\dot{G}}{A}\right) \\
\frac{\partial}{\partial \zeta}\left(\frac{\dot{G}}{A}\right)
\end{array}\right) \stackrel{!}{=} 0
$$

These optimization requirements lead to the requirements which determine the optimal dimensionless geometric parameters $a_{T, o p t}, a_{V, o p t}$ and the optimal operation parameter of the turbine $\zeta_{\text {opt }}$

$$
\left(\begin{array}{c}
\frac{\partial C_{P}}{\partial a_{T}} \frac{\varrho_{g}}{2} c^{3} \frac{\eta_{\text {Elec }} \eta_{G e n}}{H_{u, H_{2}}} p_{H_{2}} \\
\frac{\partial C_{P}}{\partial a_{V}} \frac{\varrho g}{2} c^{3} \frac{\eta_{E l e c} \eta_{G e n}}{H_{u, H_{2}}} p_{H_{2}} \\
\frac{\partial C_{P}}{\partial \zeta}
\end{array}\right)=\left(\begin{array}{c}
C R F p_{T} \\
C R F p_{V} \\
0
\end{array}\right)
$$

If all these requirements are fulfilled the system is energetically and economically optimal. This means the system cannot be improved energetically 
without worsening it economically. The sum of all systems, which fulfill these requirements, forms the Pareto-frontier.

\section{Specific design of an optimal energetic and economic energy converter}

Since the Pareto-frontier contains all optimal systems a method shall be presented that uses the abstract fundamental system optimization method to end up with a specific but optimal design. Therefore an energy task and an available vessel are defined. The energy ship should be able to deliver a turbine power of $P_{S}=20 \mathrm{~kW}$. An available vessel with a wetted area of $A_{V}=50 \mathrm{~m}^{2}$ is assumed. The necessary sail area is calculated to fulfill the energy task. Therefore further assumptions are listed in Table 1.

Table 1: Listing of the boundary conditions and the assumptions.

\section{ENERGETIC QUANTITIES ECONOMIC QUANTITIES}

\begin{tabular}{ll}
\hline \hline$A_{V}=50 \mathrm{~m}^{2}$ & $n=20$ \\
$c_{D}=0.01$ & $i=0.04$ \\
$c_{L}=1.5$ & \\
$c=10 \mathrm{~m} / \mathrm{s}$ & $p_{H_{2}}=8 € / \mathrm{kg}_{\mathrm{H}_{2}}$ \\
$\alpha_{0}=70^{\circ}$ & $p_{V}=2000 € / \mathrm{m}^{2}$ \\
$\eta_{\text {Elec }}=0.7$ & $p_{T}=50000 € / \mathrm{m}^{2}$ \\
$\eta_{\text {Gen }}=0.95$ & $p_{S t}=20000 / \mathrm{A} € / \mathrm{m}^{2}$ \\
$\eta_{T}=0.9$ & \\
$\varrho=1.2 * 10^{-3}$ & \\
$H_{u, H_{2}}=119.972 * 10^{6} \mathrm{~J} / \mathrm{kg}$ & \\
& \\
\hline
\end{tabular}

\subsection{Energetic system optimization}

First the energetic system quantity, the maximal coefficient of performance, is calculated for different sail and dimensionless turbine areas. The results are shown in Figure 2.

Maximal coefficient means that the ideal axial induction factor has been determined. It is shown in Figure 2 that the coefficient of performance increases with increasing sail area. This can be explained by the higher thrust force of the vessel which will lead to higher vessel speeds. Also for higher dimensionless turbine areas the coefficient of performance converges asymptotically to a constant value. Nevertheless, no absolute optimum of the coefficient of performance can be detected by this energetic consideration. Figure 3 shows the optimal vessel speed for the different sail areas. 


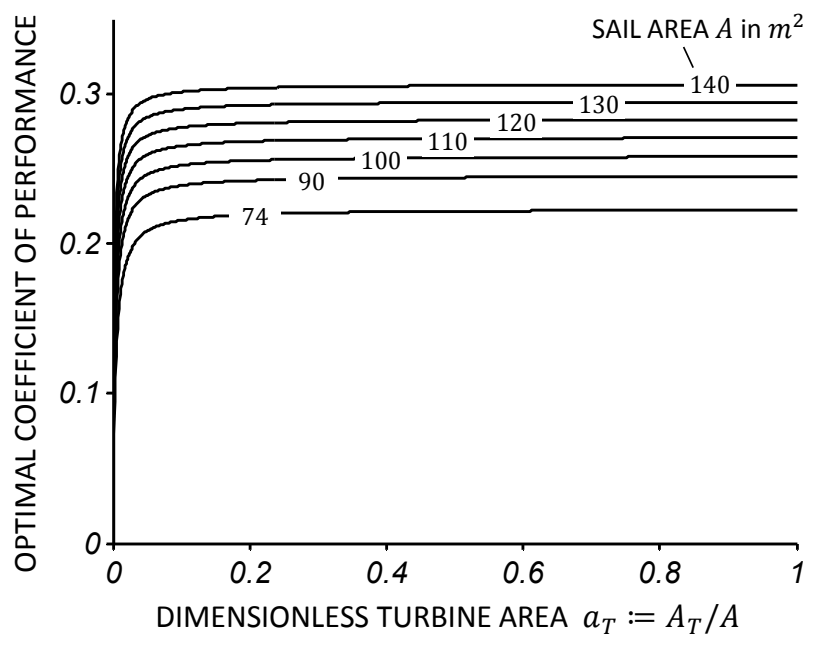

Figure 2: Maximal coefficient of performance for varying sail and turbine areas.

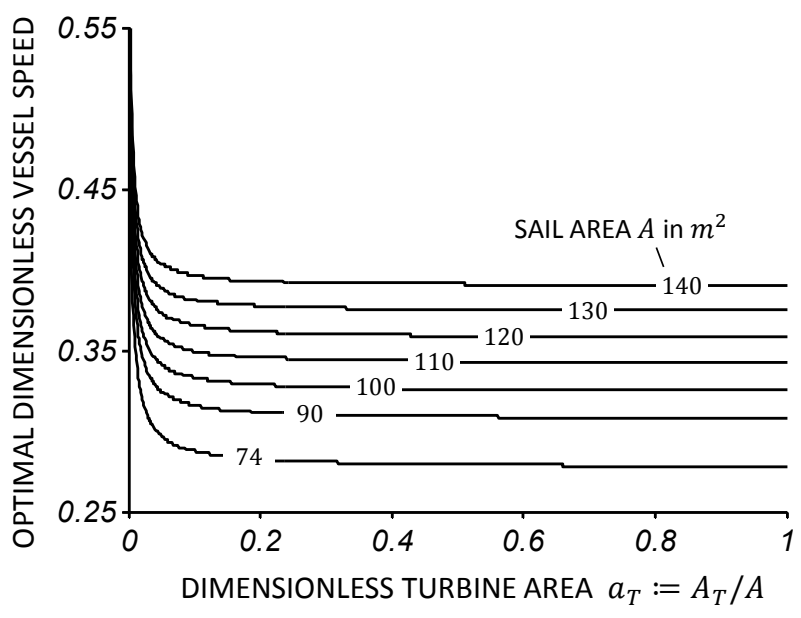

Figure 3: Optimal vessel speed for varying sail areas.

Again it can be found that the value for the optimal vessel speed converges asymptotically to a constant value. Obviously, this value depends on the sail area. The power output of the turbine increases with the sail area and increasing coefficient of performance. According to Figure 4 an energy ship with a sail area greater than $A=120 \mathrm{~m}^{2}$ can convert $20 \mathrm{~kW}$ of electrical power. By increasing the sail area the necessary turbine area will decrease. To determine the optimal sail and turbine area, economical parameters need to be considered. 


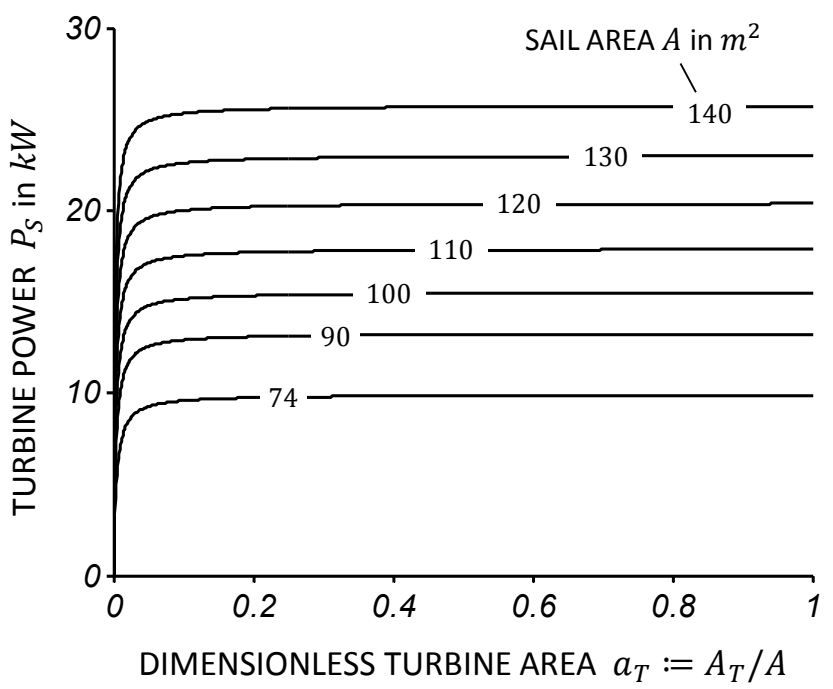

Figure 4: Maximal turbine power for varying turbine and sail areas.

\subsection{Techno-economic system optimization}

The previous calculations only considered the energetic aspect with the result that most energy can be converted if the turbine area is as large as possible. Because this is financially not feasible, economic aspects need to be considered. Therefore the results of the energetic and economic optimization shall be discussed. In Figure 5 the profit per year is shown for different dimensionless turbine areas.

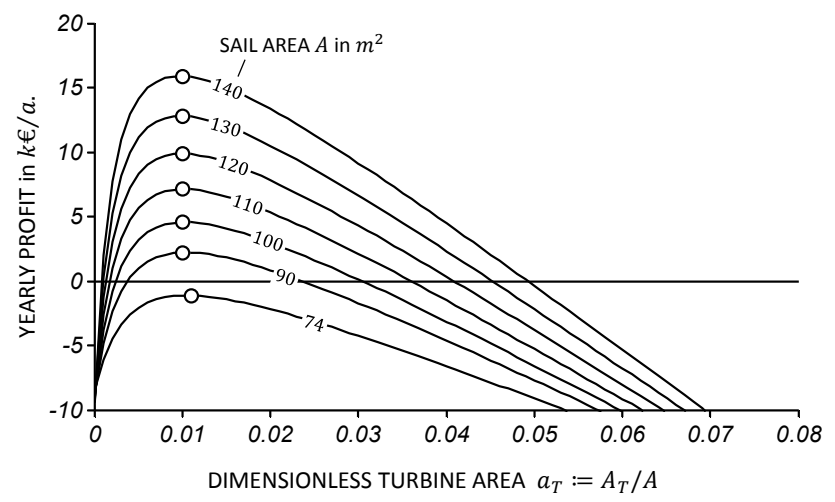

Figure 5: Yearly profit for varying sail and dimensionless turbine areas.

It is obvious that only a turbine design for positive profit is economically feasible. In the case of a sail area $A=74 \mathrm{~m}^{2}$ the yearly revenue is not large enough to exceed the yearly costs. The larger the sail area the larger is the possible turbine area to obtain a yearly profit. Nevertheless there is just one turbine area 
where the yearly profit maximizes. Those optimal turbine areas differ. This means that the optimal construction of the turbine depends on the sail area and further on every parameter that defines the yearly costs! The optimal turbine area changes for different interest rates of the capital. This means that there is also an optimal turbine area for every rate of interest. The optimal turbine diameter for the sail areas is shown in Figure 6.

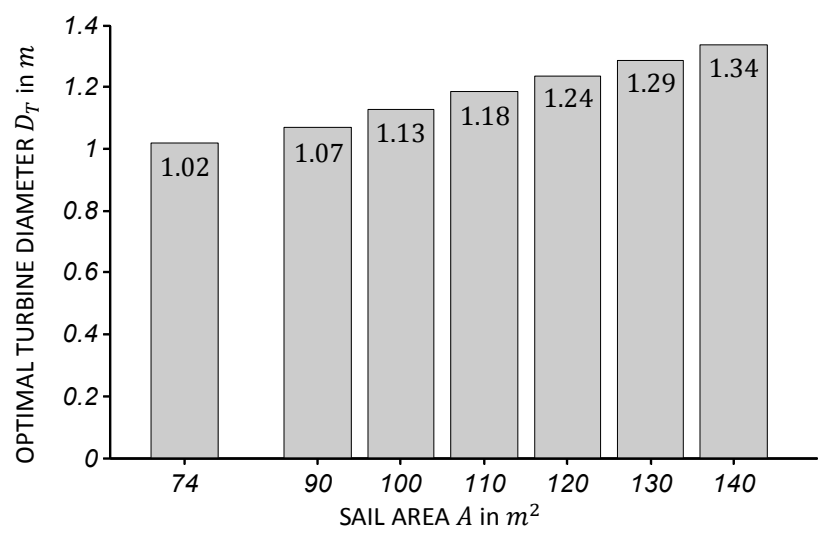

Figure 6: $\quad$ Optimal turbine diameter for varying sail areas.

Only the economic and energetic optimization provides reasonable turbine areas. Figure 7 shows the turbine power for optimal energy ships and varying sail areas.

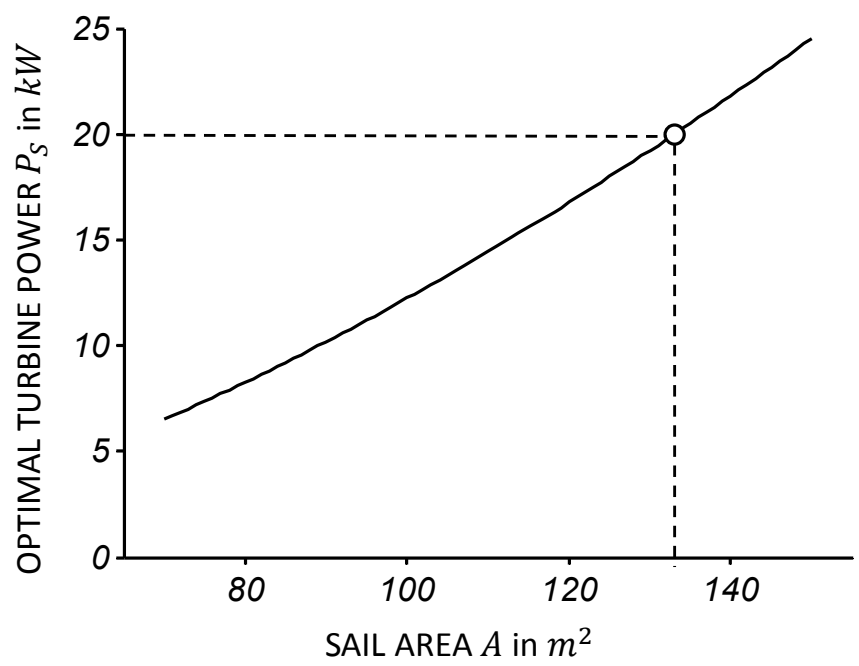

Figure 7: Turbine power and mass flow of hydrogen for optimal designed energy ships. 
For a turbine power output of $P_{S}=20 \mathrm{~kW}$ a sail area of $A=133 \mathrm{~m}^{2}$ is necessary. The optimal turbine area for this sail area is $A_{T, o p t} \approx 1.33 \mathrm{~m}^{2}$. The optimal vessel speed for this specific case is $V \approx 4.429 \mathrm{~m} / \mathrm{s}$. The optimal axial induction factor can be calculated as $\zeta_{\text {opt }}=0.782$. The control system of the energy ship needs to provide this value through the control of the generator. Finally, the expected profit per year can be calculated to $\dot{G} \approx 13.710 € / a$. The main values for the derived energetic and economic energy ship are summarized in Table 2.

Table 2: Main energetic and design parameters.

\begin{tabular}{ll}
\hline ENERGETIC PARAMETERS & DESIGN PARAMETERS \\
\hline \hline$P_{S}=20 \mathrm{~kW}$ & $A=133 \mathrm{~m}^{2}$ \\
$\zeta_{\text {opt }}=0.782$ & $A_{V}=50 \mathrm{~m}^{2}$ \\
$c=10 \mathrm{~m} / \mathrm{s}$ & $A_{T, \text { opt }}=1.33 \mathrm{~m}^{2}$ \\
$V=4.429 \mathrm{~m} / \mathrm{s}$ & \\
\hline
\end{tabular}

\section{Generalization of the method}

The above shown method to derive a specific optimal energy ship design for a given energy task is now generalized to apply it for varying requirements. The generalized method can be seen in Figure 8.

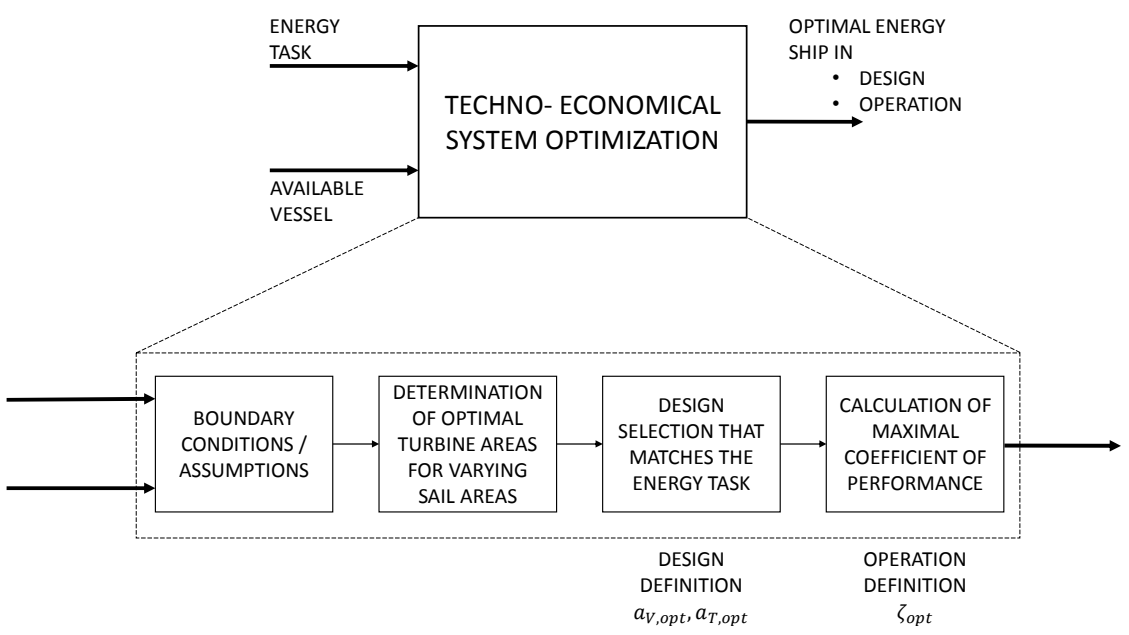

Figure 8: Generalized method for the derivation of optimal energy ships. 
The techno-economical optimization of the energy ship scenario provides the optimal energy ship design and operation. For the optimization an energy task needs to be stated. This energy task can be formulated as a necessary turbine power or a necessary mass flow of hydrogen. A vessel meeting this energy task needs to be chosen. The first step is to list the boundary conditions (e.g. the sail lift coefficient) and the assumptions (e.g. area specific turbine prices). The next step is to use the techno-economical optimization to determine the optimal turbine area for varying sail areas. The definition of the design parameters is accomplished by matching the power output of the energy ship to the energy task. In this way the optimal dimensionless geometric parameters $a_{V, o p t}$ and $a_{T, o p t}$ are defined. The last step considers the operation of the energy ship. To ensure maximal energetic efficiency for the defined design the coefficient of performance needs to be calculated. The coefficient of performance becomes a maximum for the optimal induction factor $\zeta_{\text {opt }}$ which defines the operation of the vessel. The optimal vessel speed can be calculated using the optimal axial induction factor.

\section{Conclusion}

By applying the abstract and fundamental description and optimization of the energy ship scenario it is shown how to determine an optimal specific design for a given energy task. It is found that the system design needs to consider more than the energetic efficiency. The differences in designing an energy ship by considering energetic aspects only and the combined energetic and economic aspects are demonstrated. The optimization leads to the optimal energy ship in design and operation. The specific system design is part of the Pareto-frontier, which describes the amount of all optimal energy systems.

\section{Acknowledgement}

The authors would like to thank the Deutsche Forschungsgemeinschaft (DFG) for the financial support in the framework of the Excellence Initiative, Darmstadt Graduate School of Excellence Energy Science and Engineering (GSC 1070).

\section{References}

[1] R.E. Salomon, Process of converting wind energy to elemental hydrogen and apparatus therefor, US Patent 4,335,093, June 15, 1982

[2] M. F. Platzer, N. Sarigul-Klijn, A novel approach to extract power from freeflowing water and high-altitude jet streams, ASME Paper No. ES 200990146, June 2009

[3] M. F. Platzer, N. Sarigul-Klijn, J. Young, M.A. Ashraf, J.C.S. Lai, Renewable hydrogen production using sailing ships, ASME Journal of Energy Resources Technology, Vol. 136, June 2014 
[4] M. Platzer, M. Lennie, D. Vogt, Analysis of the conversion of ocean wind power into hydrogen, Proc. World Renewable Energy Congress, Perth, Australia, July 2013

[5] J. Kim, C. Park, Wind power generation with a parawing on ships, a proposal, Journal of Energy, Vol. 35, pp. 1425-1432, 2010

[6] P. Pelz, M. Holl, M.F. Platzer, Analytical method towards an optimal energetic and economical ocean-wind energy converter, to be submitted to Journal of Renewable Energy, 2014

[7] A. Betz, Das Maximum der theoretisch möglichen Ausnützung des Windes durch Windmotoren, Zeitschrift für das gesamte Turbinenwesen, 1920 